\title{
Instability of channel flow of a shear-thinning White-Metzner fluid
}

\author{
H.J. Wilson ${ }^{1}$, J.M. Rallison ${ }^{*}$ \\ Department of Applied Mathematics and Theoretical Physics, University of Cambridge, \\ Silver Street, Cambridge CB3 9EW, UK
}

Received 9 November 1998

\begin{abstract}
We consider the inertialess planar channel flow of a White-Metzner (WM) fluid having a power-law viscosity with exponent $n$. The case $n=1$ corresponds to an upper-convected Maxwell (UCM) fluid. We explore the linear stability of such a flow to perturbations of wavelength $k^{-1}$. We find numerically that if $n<n_{\mathrm{c}} \approx 0.3$ there is an instability to disturbances having wavelength comparable with the channel width. For $n$ close to $n_{\mathrm{c}}$, this is the only unstable disturbance. For even smaller $n$, several unstable modes appear, and very short waves become unstable and have the largest growth rate. If $n$ exceeds $n_{\mathrm{c}}$, all disturbances are linearly stable. We consider asymptotically both the long-wave limit which is stable for all $n$, and the shortwave limit for which waves grow or decay at a finite rate independent of $k$ for each $n$.

The mechanism of this elastic shear-thinning instability is discussed. (C) 1999 Elsevier Science B.V. All rights reserved.
\end{abstract}

Keywords: Channel flow; Instability; Long wave; Short wave; Normal stresses; Shear-thinning; Power-law; White-Metzner; UCM

\section{Introduction}

As catalogued by Larson [1], instabilities of flows of polymeric fluids are legion. They are important for their own fundamental scientific interest, providing a severe test of constitutive modelling, and also, of course, because of their relevance to processing applications.

In this paper, we focus on the simplest flow geometry, sketched in Fig. 1, an incompressible rectilinear channel flow having negligible inertia and no-slip boundary conditions. If a flow of this kind is unstable, it provides a fertile ground on which observed extrudate distortions (e.g. melt fracture) may develop.

\footnotetext{
* Corresponding author.

${ }^{1}$ Current address: Department of Chemical Engineering, University of Colorado, Boulder, CO 80309-0424, USA.
} 


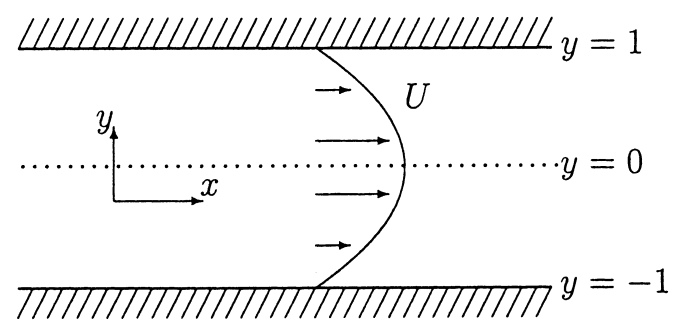

Fig. 1. Flow geometry.

In an earlier paper [2] we discussed pure elastic (i.e. inertialess) co-extrusion instabilities of a class of Oldroyd-B fluids in channel flow. We demonstrated that two ingredients are required to sustain such an instability: first, there must be a sufficiently steep gradient in normal stress within the flow, and second, this gradient must result from a rapid change in a material property that is convected by the fluid. The mechanism for this instability is elucidated in [2,3]; it relies on the tilting of base flow streamlines to expose the rapid $N_{1}$ variation, but by itself this is insufficient. For a particular choice of flow parameters a WM fluid having rapid variation of relaxation time with shear rate is shown in [2] to remain stable, while an Oldroyd-B fluid with the same base state velocity and stress profiles is unstable.

These considerations, however, do not rule out the possibility of other instability mechanisms, and in this paper we consider a shear-thinning WM fluid having constitutive equation [4]

$$
\boldsymbol{\sigma}=-p \boldsymbol{I}+G_{\mathrm{M}} \boldsymbol{A},
$$

where

$$
\stackrel{\nabla}{\boldsymbol{A}}=-\frac{1}{\tau(\dot{\gamma})}(\boldsymbol{A}-\boldsymbol{I}) \quad \text { and } \quad \dot{\gamma}=\sqrt{2 \boldsymbol{E}: \boldsymbol{E}},
$$

with $\mathbf{E}$ the rate of strain tensor, and $G_{\mathrm{M}}$ a (constant) elastic modulus of the material. The physical idea underlying this model is that the polymer relaxation time $\tau$ should depend instantaneously upon the local velocity gradient $\dot{\gamma}$. In steady, simple shear it gives a viscosity $G_{\mathrm{M}} \tau(\dot{\gamma})$ and first normal stress difference $G_{\mathrm{M}} \tau^{2}(\dot{\gamma}) \dot{\gamma}^{2}$.

If $\tau$ is constant, we recover the UCM model whose stability characteristics are well known [5], and this will provide a useful check on our computations. To explore shear-thinning possibilities we put

$$
\tau(\dot{\gamma})=K_{\mathrm{M}} \dot{\gamma}^{n-1}
$$

where $K_{\mathrm{M}}$ and $n \in[0,1]$ are material constants. The shear viscosity is then proportional to $\dot{\gamma}^{n-1}$, so the fluid shear-thins, and the normal stress is proportional to $\dot{\gamma}^{2 n}$.

Apart from the UCM case $(n=1)$ this power-law dependence gives a weakly singular behaviour near $\dot{\gamma}=0$. We could remedy this difficulty by making a different choice (e.g. a Bird-Carreau viscosity [6]) but to do so would necessarily introduce a new dimensionless group into an already complicated problem.

For the channel flow sketched in Fig. 1, it is convenient to scale lengths by the channel half-width $L$, velocities by the centreline velocity $U_{0}$, times by the average shear rate in the channel, $L / U_{0}$, and 
stresses by $G_{\mathrm{M}} K_{\mathrm{M}}\left(U_{0} / L\right)^{n}$, giving a non-dimensional constitutive equation:

$$
\begin{aligned}
& \boldsymbol{\sigma}=-p \boldsymbol{I}+\frac{1}{W} \boldsymbol{A}, \\
& \stackrel{\nabla}{\boldsymbol{A}}=-\frac{1}{W \dot{\gamma}^{n-1}}(\boldsymbol{A}-\boldsymbol{I}),
\end{aligned}
$$

where the channel Weissenberg number $W=K_{\mathrm{M}}\left(U_{0} / L\right)^{n}$.

We shall consider only incompressible inertialess flows for which $\boldsymbol{\nabla} \cdot \boldsymbol{u}=0$ and $\boldsymbol{\nabla} \cdot \boldsymbol{\sigma}=0$. There are thus two parameters at our disposal in describing the base state: the degree of shear-thinning $n$ and the flow strength $W$.

In the following section we derive the steady velocity and stress profiles for this fluid for a pressure driven channel flow, and then consider the evolution of an infinitesimal perturbation to the flow having wave number $k$ (scaled by $L$ ) and complex frequency $\omega$ (scaled by $L / U_{0}$ ). The stability characteristics are controlled by the sign of the imaginary part of $\omega(k)$. A full investigation of the stability problem therefore involves consideration of three parameters $W, n$ and $k$.

Some simplification is possible for long waves $(k \rightarrow 0$, see Section 3) and for short waves $(k \rightarrow \infty$, Section 4), and these provide checks for a numerical code for general $k$, whose results are presented in Section 5. We find that if the fluid shear-thins sufficiently, then the flow is unstable to disturbances having moderate wavelength. We offer a description of the physical mechanism for this elastic shearthinning instability in Section 6.

\section{Governing equations for a channel flow}

\subsection{Base flow}

Solving the steady momentum equation we find the base flow

$$
U(y)=1-y^{(n+1) / n}
$$

The remaining base state variables are then

$$
\begin{aligned}
& \dot{\gamma}=\left|U^{\prime}\right|=-U^{\prime}=\left(\frac{n+1}{n}\right) y^{1 / n}, \\
& \tau=W\left(\frac{n+1}{n}\right)^{n-1} y^{(n-1) / n}, \\
& \boldsymbol{A}=\left(\begin{array}{ll}
1+2\left(\frac{n+1}{n}\right)^{2 n} W^{2} y^{2} & -\left(\frac{n+1}{n}\right)^{n} W y \\
-\left(\frac{n+1}{n}\right)^{n} W y & 1
\end{array}\right),
\end{aligned}
$$




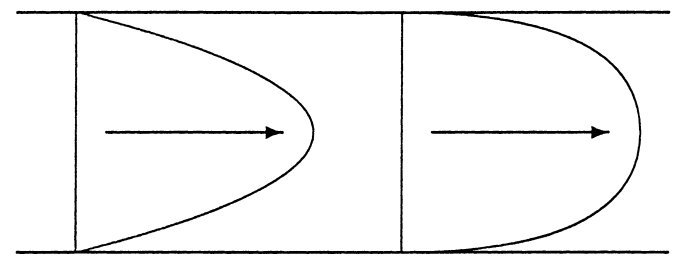

Fig. 2. Base-flow velocity profile for two values of $n$. On the left is the parabolic profile for $n=1$ (a UCM fluid), while a shear-thinning fluid with $n \ll 1$ is on the right.

with the pressure given to within an additive constant as

$$
P=\frac{1}{W}+G x
$$

where $G$ is the pressure gradient, having the value dictated by our normalisation of $U$ :

$$
G=-\left(\frac{n+1}{n}\right)^{n} \text {. }
$$

The dependence of $G$ on $n$ is weak: $G=-2$ when $n=1 ; G=-1$ when $n=0$. The base-state stress is

$$
\boldsymbol{\Sigma}=\left(\begin{array}{lc}
-G x+2 W G^{2} y^{2} & G y \\
G y & -G x
\end{array}\right) .
$$

The velocity $U$ is sketched in Fig. 2 for two values of $n$. For $n=1$ the profile is parabolic, because the time constant $\tau$ and hence the viscosity are uniform across the channel. For small $n$ on the other hand, the profile is close to a plug flow with $\dot{\gamma}$ very small and $\tau$ correspondingly large across most of the channel, together with boundary layers of width $n$ near $y= \pm 1$ within which $\dot{\gamma}$ is large (of size $n^{-1}$ ) and $\tau$ correspondingly small. We note that the channel Weissenberg number $W$ is nevertheless representative of a local Weissenberg number $\dot{\gamma} \tau$ at all points of the channel. For $n \neq 1$, there is a weak singularity in $U(y)$ at $y=0$ resulting from the non-analyticity of the power-law $\tau(\dot{\gamma})$.

Although the normal stress thins in $\dot{\gamma}$, the shear rate near the walls is higher and so the normal stress profile $N_{1}(y)$ across the channel is, for any choice of $n$, parabolic, being largest in the high shear rate region near the walls.

\subsection{Perturbation equations}

We impose a linear perturbation on the base state in which all quantities are proportional to $\mathrm{e}^{\mathrm{ikx}-\mathrm{i} \omega \mathrm{t}}$. The wave number $k$ is taken to be real, but the frequency $\omega$ may be complex. The imaginary part of $\omega$ is then the growth rate, and the real part represents advection of the perturbation along the channel. The incompressible perturbation velocity field admits a stream function, $\psi(y)$ so that

$$
u=\psi^{\prime} \quad \text { and } \quad v=-\mathrm{i} k \psi
$$

where the prime denotes differentiation with respect to $y$. 
The linearised momentum equations for the perturbation stress $\sigma$ and pressure $p$ are

$$
\begin{aligned}
& \mathrm{i} k \sigma_{11}+\sigma_{12}^{\prime}=0, \\
& \mathrm{i} k \sigma_{12}+\sigma_{22}^{\prime}=0, \\
& \sigma_{11}=-p+\frac{1}{W} a_{11}, \\
& \sigma_{12}=\frac{1}{W} a_{12}, \\
& \sigma_{22}=-p+\frac{1}{W} a_{22},
\end{aligned}
$$

in which $\boldsymbol{a}$ is the perturbation to $\boldsymbol{A}$. The pressure $p$ may be eliminated from Eqs. (1)-(5) to give instead a single vorticity equation:

$$
a_{12}^{\prime \prime}+k^{2} a_{12}+\mathrm{i} k\left(a_{11}^{\prime}-a_{22}^{\prime}\right)=0 .
$$

The evolution equations for the perturbations $\boldsymbol{a}$ are

$$
\begin{aligned}
& \left(-\mathrm{i} \omega+\mathrm{i} k U+\frac{1}{\tau}\right) a_{11}=\mathrm{i} k \psi A_{11}^{\prime}+2 A_{12} \psi^{\prime \prime}+2 A_{11} \mathrm{i} k \psi^{\prime}+2 U^{\prime} a_{12}+\frac{\Delta \tau}{\tau^{2}}\left(A_{11}-1\right), \\
& \left(-\mathrm{i} \omega+\mathrm{i} k U+\frac{1}{\tau}\right) a_{12}=\mathrm{i} k \psi A_{12}^{\prime}+\psi^{\prime \prime}+A_{11} k^{2} \psi+U^{\prime} a_{22}+\frac{\Delta \tau}{\tau^{2}} A_{12} \\
& \left(-\mathrm{i} \omega+\mathrm{i} k U+\frac{1}{\tau}\right) a_{22}=-2 \mathrm{i} k \psi^{\prime}+2 A_{12} k^{2} \psi
\end{aligned}
$$

where $\tau$ is the relaxation time associated with the base state

$$
\tau=W\left(\frac{n+1}{n}\right)^{n-1} y^{(n-1) / n},
$$

and $\Delta \tau$ is its perturbation arising from the changed value of $\dot{\gamma}$ :

$$
\Delta \tau=-W(n-1)\left(\frac{n+1}{n}\right)^{n-2} y^{(n-2) / n}\left(\psi^{\prime \prime}+k^{2} \psi\right) .
$$

At this point, we may exploit the up-down symmetry of the base flow to consider separately sinuous models ( $\psi$ even) and varicose modes ( $\psi$ odd). For the sinuous case, however, the Taylor series

$$
\tau(\dot{\gamma}+\Delta \dot{\gamma})=W(\dot{\gamma}+\Delta \dot{\gamma})^{n-1} \approx W \dot{\gamma}^{n-1}+(n-1) W \dot{\gamma}^{n-2} \Delta \dot{\gamma}
$$

breaks down near the centre line as a result of the singular behaviour of $\tau(\dot{\gamma})$ at $\dot{\gamma}=0$. $^{2}$ For a varicose mode however, the perturbation to the shear rate, $\Delta \dot{\gamma}$, proportional to $\psi^{\prime \prime}+k^{2} \psi$, vanishes where $y=0$,

\footnotetext{
${ }^{2}$ It would, of course, be possible to treat sinuous modes by applying a numerical patch near $y=0$, but the results for $\omega$ would then depend unsatisfactorily upon the size of the patch. Alternatively, as noted in Section 1, a Bird-Carreau regularisation of the viscosity could be employed.
} 
and thus the linearisation is rescued, and the system of equations has a removable singularity at $y=0$. In what follows, therefore, we focus only on varicose modes for which

$$
\psi(0)=\psi^{\prime \prime}(0)=0 \text {. }
$$

Finally the boundary conditions at the wall are

$$
\psi(1)=\psi^{\prime}(1)=0 .
$$

Our aim is to determine the complex eigenvalue $\omega(k)$ for a nontrivial solution of the Eqs. (6)-(11) subject to the boundary conditions (12) and (13).

It is notoriously easy to generate spurious numerical instabilities from sets of equations of this kind. We therefore start by constructing asymptotic approximations against which our numerics can be checked. In addition, the availability of solutions for $n=1$ provides a further benchmark and a useful starting point for parameter continuation.

\section{Long waves}

In the long-wave limit $k \rightarrow 0$ we expand in powers of $k$. The leading- and first-order calculations are summarised here. We write

$$
\begin{aligned}
& \omega=\omega_{0}+k \omega_{1}+\mathrm{O}\left(k^{2}\right), \\
& \psi=\psi_{0}+k \psi_{1}+\mathrm{O}\left(k^{2}\right) .
\end{aligned}
$$

\subsection{Leading order calculation}

In the long-wave limit, Eq. (6) shows that for a varicose mode the perturbation shear stress $a_{12}$ is linear in $y$. Eq. (8) then gives to within an arbitrary multiplicative constant

$$
\psi_{0}^{\prime \prime}=-\mathrm{i} \omega_{0} y+\frac{1}{W}\left(\frac{n+1}{n}\right)^{n-1} y^{1 / n}
$$

Integrating, and applying the boundary conditions $\psi_{0}^{\prime}(1)=0$, and $\psi_{0}(0)=0$ gives

$$
\psi_{0}=-\mathrm{i} \omega_{0}\left(\frac{1}{6} y^{3}-\frac{1}{2} y\right)+\frac{1}{W}\left(\frac{n}{n+1}\right)^{n} \frac{n y^{(2 n+1) / n}-(2 n+1) y}{2 n+1} .
$$

Finally the condition $\psi_{0}(1)=0$ yields

$$
\omega_{0}=-\frac{i}{W}\left(\frac{n}{n+1}\right)^{n-1} \frac{3 n}{2 n+1} .
$$

It follows that infinitely long waves $(k \rightarrow 0)$ are stable for all $n$ and $W$. In the case $n=1$, this eigenvalue is the pure relaxation mode $\omega_{0} \rightarrow-\mathrm{i} / W$ for which $\psi_{0} \rightarrow 0$. 


\subsection{Order-k calculation}

When the leading-order quantities are substituted into the first-order expansion in small $k$, the result is a third-order differential equation for $\psi_{1}$. The details are presented in [7]. A surprising simplification is that $W$ may be scaled out of the $\mathrm{O}(k)$ problem, showing that $\omega_{1}$ is independent of $W$ and thus depends upon $n$ alone. It follows that there exists a mode for which

$$
\omega=-\frac{\omega_{0}(n)}{W}+k \omega_{1}(n)+\mathrm{O}\left(k^{2}\right)
$$

and hence a necessary condition for instability is that $k W$ should be sufficiently large. We have not been able to find an analytic solution for $\omega_{1}$, except for the Maxwell limit, $n=1$, where

$$
\omega_{1}=\left\{\begin{array}{l}
0.29039769 \pm 0.05012283 \mathrm{i} \\
0.89882760 \pm 0.12682973 \mathrm{i}
\end{array}\right.
$$

as discussed in [8]. These results provide a useful starting point for parameter continuation in $k$ as discussed below.

\subsection{Long waves and low $n$}

For a highly shear-thinning fluid in the singular limit where $n \rightarrow 0$, some analytic progress can be made. We consider the joint limit for long waves in which $k \rightarrow 0$ and $n \rightarrow 0$, with the ratio $\lambda^{2}=k W / 2 n$ constant. We assume that $\omega$ will be an order 1 quantity, and check this a posteriori.

At leading order the perturbation shear stress $a_{12}$ is again linear in $y$ and hence, from Eq. (8),

$$
n \psi^{\prime \prime}-2 \mathrm{i} n \lambda^{2} \psi=-\mathrm{i} \omega y+\frac{1}{n W} y^{1 / n}
$$

A solution at leading order in small $n$ is

$$
\psi=\frac{\omega}{2 \lambda^{2}} y+\frac{n y^{1 / n}}{W}+B \sinh [(1+\mathrm{i}) \lambda y]
$$

for some constant $B$, where we have applied the condition that $\psi(0)=0$. The wall boundary conditions, $\psi(1)=0$ and $\psi^{\prime}(1)=0$, then determine $B$ and give

$$
\omega=\frac{1}{W} \frac{2 \lambda^{2}}{(1+\mathrm{i}) \lambda \operatorname{coth}[(1+\mathrm{i}) \lambda]-1} .
$$

In the limit $\lambda \rightarrow 0$, i.e. $k \rightarrow 0$ for small finite $n$, this becomes

$$
\omega=-\frac{3 \mathrm{i}}{W}
$$

which is indeed the $n \rightarrow 0$ limit of the leading-order expression for $k \rightarrow 0$, given by Eq. (14). For all values of $\lambda$, Eq. (16) shows that the flow is stable. The streamlines of the perturbation flow are shown for one representative case in Fig. 3. 


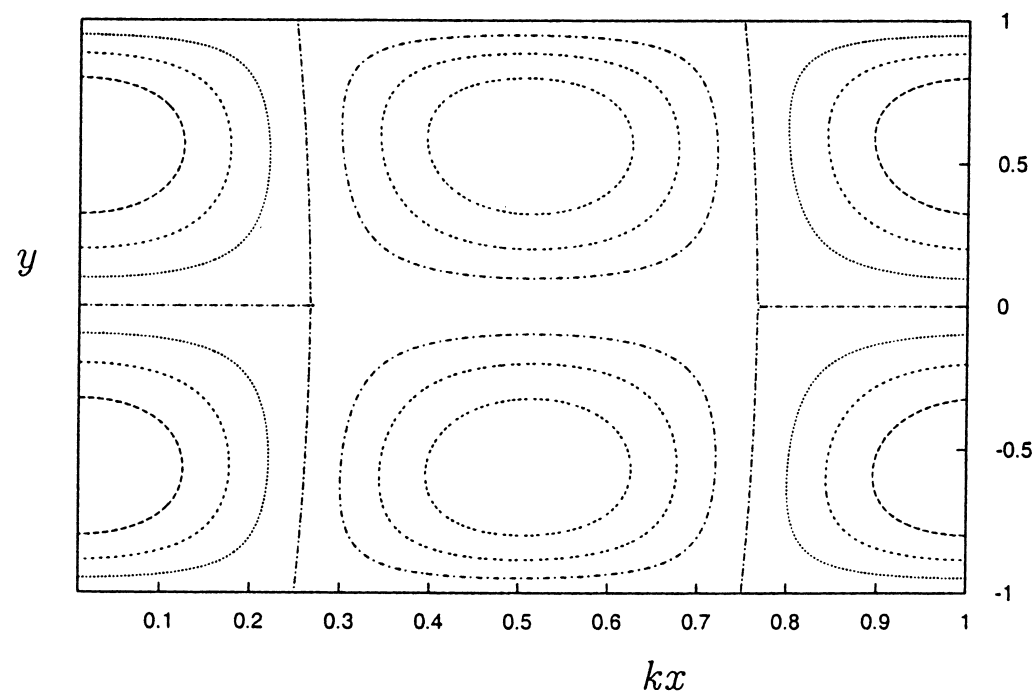

Fig. 3. Streamlines of the stable perturbation flow (15), for the asymptotic limit of low $n$ and low $k$. The parameter values are $n=k=0.01, W=2$.

Unfortunately, this result is of limited usefulness in checking our code, because the asymptotic behaviour is achieved only for very small values of $n$ for which difficulties arise in obtaining a numerical solution to the full problem.

\section{Short waves}

\subsection{Scaling for short waves}

A WM fluid with a power-law relaxation function has no intrinsic length-scale, like the Maxwell fluid to which it specialises. The only length-scales in the problem are the channel width, normalised to unity, and the wavelength, $k^{-1}$. For very short waves (shorter than the boundary layer thickness $n$, if $n$ is small), all the lengths in the problem must scale with $k^{-1}$, and so the disturbance is localised at some point in the channel.

While, in principle, such a mode could be localised anywhere, the distinguished points are the centre of the channel, $y=0$, and the wall, $y=1$. We concentrate on wall modes here. The relevant timescale for growth or decay of such a perturbation must be determined by the wall shear-rate, $\dot{\gamma}_{w}=(n+1) / n$, rather than the average shear rate in the channel which was the basis of our non-dimensionalisation. These differ by a factor of $n$ if $n$ is small.

We let $\eta=k(y-1)$, d denote $\partial / \partial \eta$, and take the limit $k^{-1} \rightarrow 0$. We find it convenient to rescale $\boldsymbol{a}$ by a factor of $k^{2} \dot{\gamma}_{w}^{-1}$, and to define $\mathscr{W}=W((n+1) / n)^{n}$. The quantity $\mathscr{W}$ is identical to $W$ as $n \rightarrow 0$ and differs from $W$ by a factor of 2 as $n \rightarrow 1$. With these modifications the perturbation equations become

$$
\left(-\mathrm{i} \omega \dot{\gamma}_{w}^{-1}-\mathrm{i} \eta+\frac{1}{\mathscr{W}}\right) a_{11}=2 \mathrm{i}\left(1+2 \mathscr{W}^{2}\right) \mathrm{d} \psi-2 \mathscr{W} n \mathrm{~d}^{2} \psi-2 a_{12}-2 \mathscr{W}(n-1) \psi
$$




$$
\begin{aligned}
& \left(-\mathrm{i} \omega \dot{\gamma}_{w}^{-1}-\mathrm{i} \eta+\frac{1}{\mathscr{W}}\right) a_{12}=\left(1+2 \mathscr{W}^{2}\right) \psi+n \mathrm{~d}^{2} \psi-a_{22}+(n-1) \psi \\
& \left(-\mathrm{i} \omega \dot{\gamma}_{w}^{-1}-\mathrm{i} \eta+\frac{1}{\mathscr{W}}\right) a_{22}=-2 \mathscr{W} \psi-2 \mathrm{id} \psi \\
& \left(\mathrm{d}^{2}+1\right) a_{12}+\mathrm{id}\left(a_{11}-a_{22}\right)=0
\end{aligned}
$$

with boundary conditions

$$
\psi(0)=\mathrm{d} \psi(0)=0, \quad \text { and } \quad \psi \rightarrow 0 \text { as } \eta \rightarrow \infty
$$

As anticipated, $k$ has disappeared from the problem. Thus, the growth or decay of short waves is independent of wavelength as $k \rightarrow \infty$. Furthermore, it is the product $\omega \dot{\gamma}_{w}^{-1}$ that arises naturally, reflecting the changed timescale of the perturbation to the flow.

These equations are very similar to those for Couette flow of a UCM fluid [5]. In fact, the only difference is in the coefficient of the highest derivative, and the final term in Eqs. (17) and (18). Both these changes come from the perturbation $\Delta \tau$ caused by the infinitesimal change in effective shear-rate. The equations are, of course, those for the UCM fluid when $n=1$.

For $n=1$ we have $\dot{\gamma}_{w}=2, \mathscr{W}=2 W$, and using the eigenfunctions of Gorodtsov and Leonov [5], the leading-order eigenvalue as $k \rightarrow \infty$ is

$$
\omega \dot{\gamma}_{w}^{-1}=\frac{-1}{1-\beta-\mathrm{i} \mathscr{W}}
$$

where $\beta=1\left(1+\mathscr{W}^{2}\right)^{1 / 2}>0$. This root is stable for all values of $\mathscr{W}$.

\subsection{Numerical results for short waves}

In the short-wave limit, a numerical study reveals only one mode with the wall-boundary-layer scaling outlined above. This root is given by Eq. (21) in the limit $n=1$, and may be continued to lower $n$. Two cases $(W=1$ and $W=2)$ are shown in Figs. 4 and 5.

We see that for any fixed value of $W$, the mode remains stable as $n$ decreases, until $n$ becomes small $(n<0.2)$, when instability may occur, depending on the value of $W$. Thus sufficiently short waves can grow, though at finite rate for any fixed $n$.

Examples of the stream functions for a stable and an unstable case are shown in Fig. 6.

Fig. 7 shows the perturbation streamlines for the unstable mode at $W=2, n=0.15$. Numerical difficulties occur for smaller $n$, because of the large values of $\omega$ and the high shear-rates involved. We note however that as $n \rightarrow 0$, if $W$ is fixed, $\omega \dot{\gamma}_{w}^{-1}$ should remain of order unity in Eqs. (17)-(19). In consequence $\omega \sim n^{-1}$ as $n \rightarrow 0$.

This is a new instability. It differs from the co-extrusion instability [4,9]; and since the base flow has straight streamlines, it is not one of the Taylor-Couette instabilities discussed by Shaqfeh [10]. We discuss its mechanism in Section 6. 


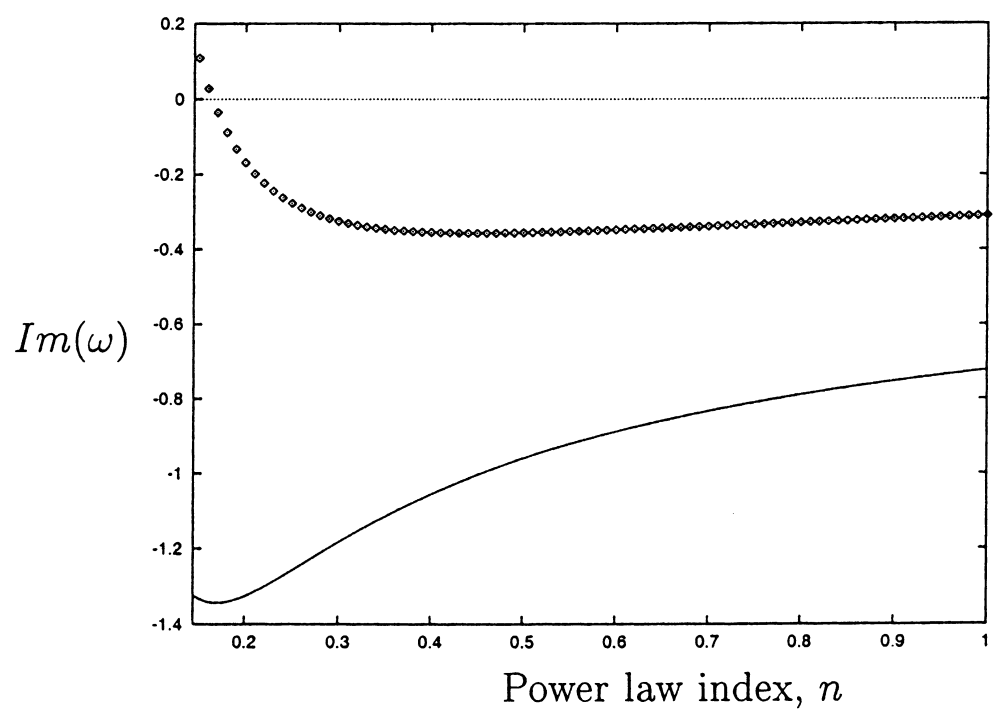

Fig. 4. Growth rate of short-wave disturbances, plotted against power law index $n$ for fixed $W=1$ (solid line) and $W=2$ (points). The mode remains stable as long as $n$ is moderate, but instability is observed for $W=2$ and $n<0.2$. The values at $n=1$ correspond with Eq. (21).

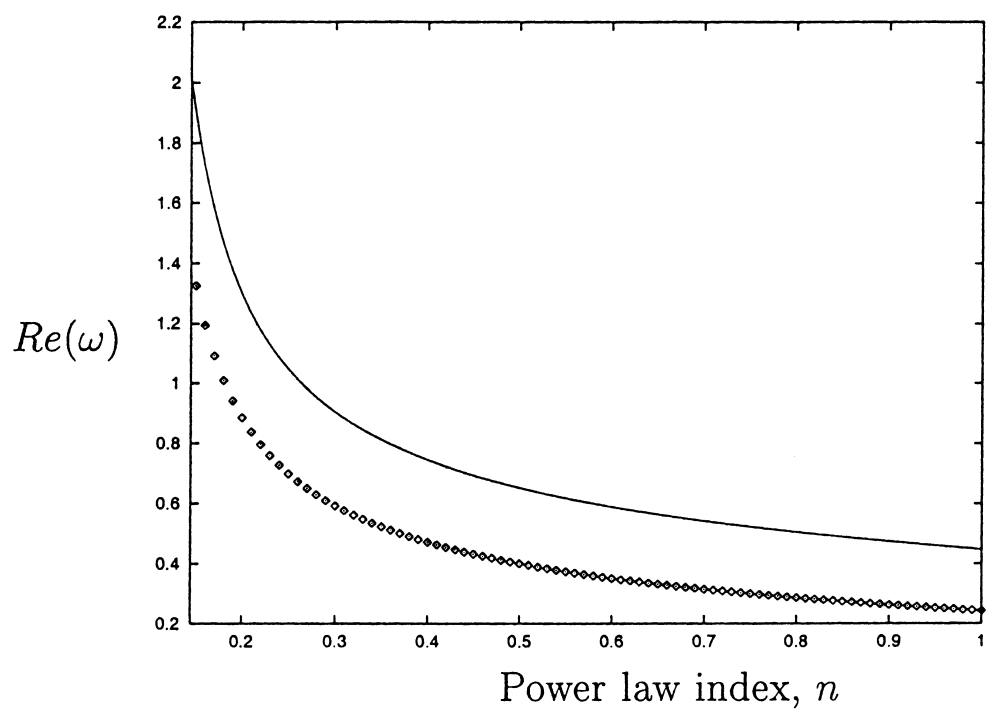

Fig. 5. Real part of the eigenvalues shown in Fig. 4.

\section{Intermediate wavelengths: numerical results}

We solve the eigenvalue problem for general $n$ using the numerical method of Ho and Denn [11]. The aim is to follow the behaviour of roots of $\omega$ in the complex plane as $n$ (or $k$ or $W$ ) varies. As a first step, we consider the modes of the UCM fluid $(n=1)$ for fixed $W(=2)$ as $k$ varies. 


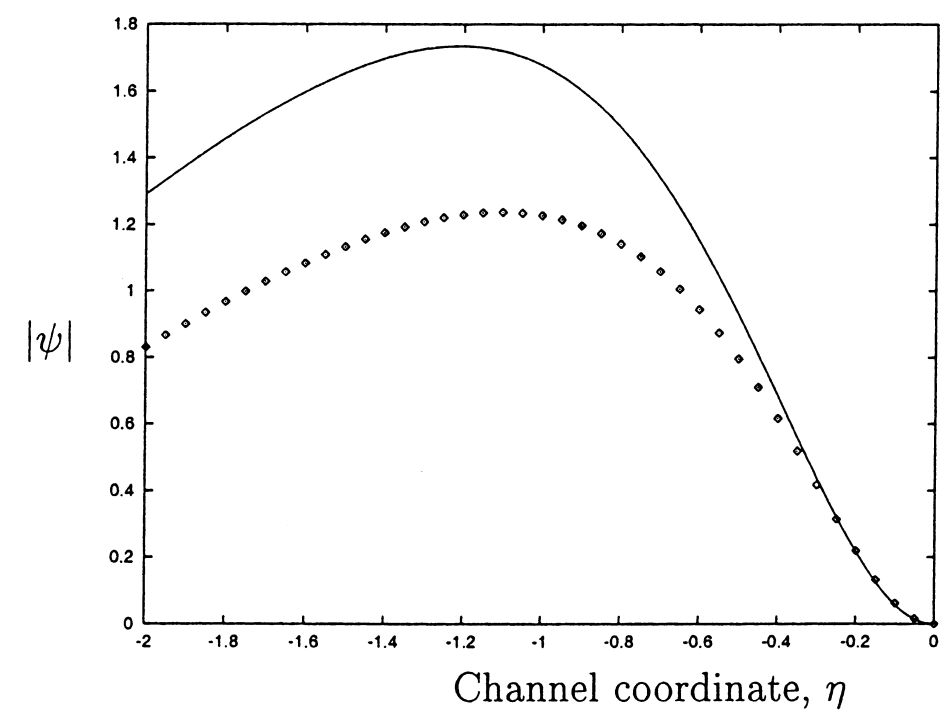

Fig. 6. The modulus of the complex streamfunction, $|\psi|$, plotted across the boundary-layer. Both results are for $n=0.15$. The solid lines are for a stable case, $W=1$, and the points for an unstable case, $W=2$.

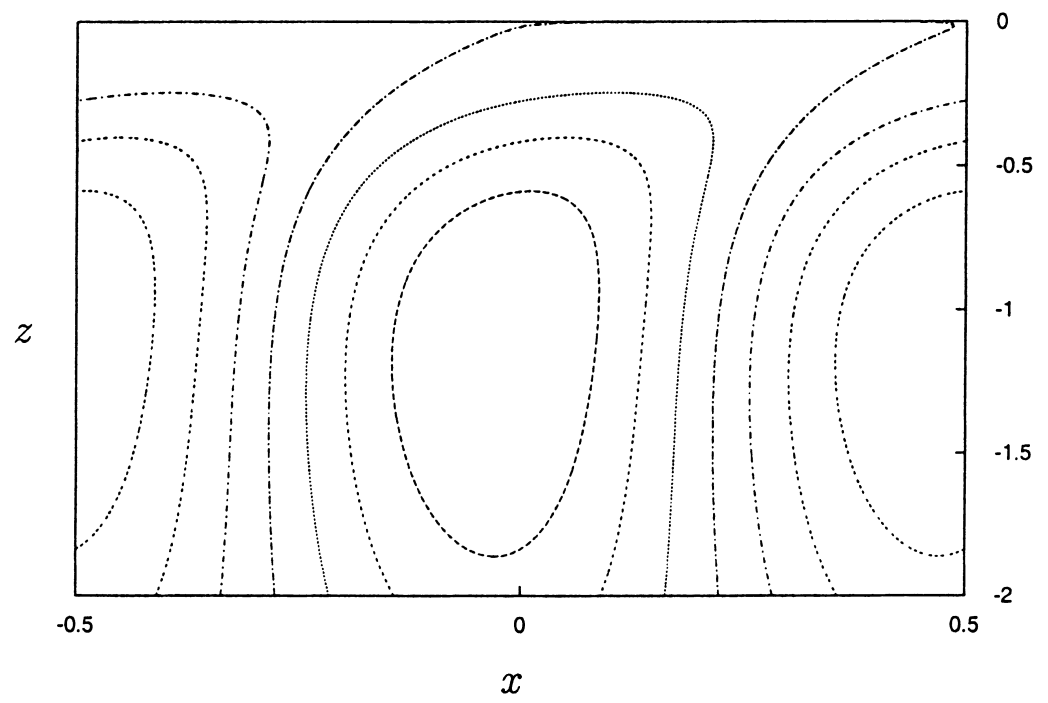

Fig. 7. Streamlines of the unstable streamfunction from Fig. 6. The disturbance decays away from the wall (at the top of the picture). $W=2, n=0.15$.

For $n=1$, long waves show four stable roots, all coalescing at $k=0$. These four roots may be continued into the regime of finite $k$. As Fig. 8 shows, one $\operatorname{root}(\operatorname{root} \mathrm{A})$ ceases to exist at $k \approx 0.4$. This is because the equations have a continuous spectrum of stable modes that correspond to a branch cut in the $\omega$-plane. This is a non-analytic region, and so analytic continuation is invalid. The disappearing root enters the continuous spectrum at the branch point, where $k \approx 0.4$. 


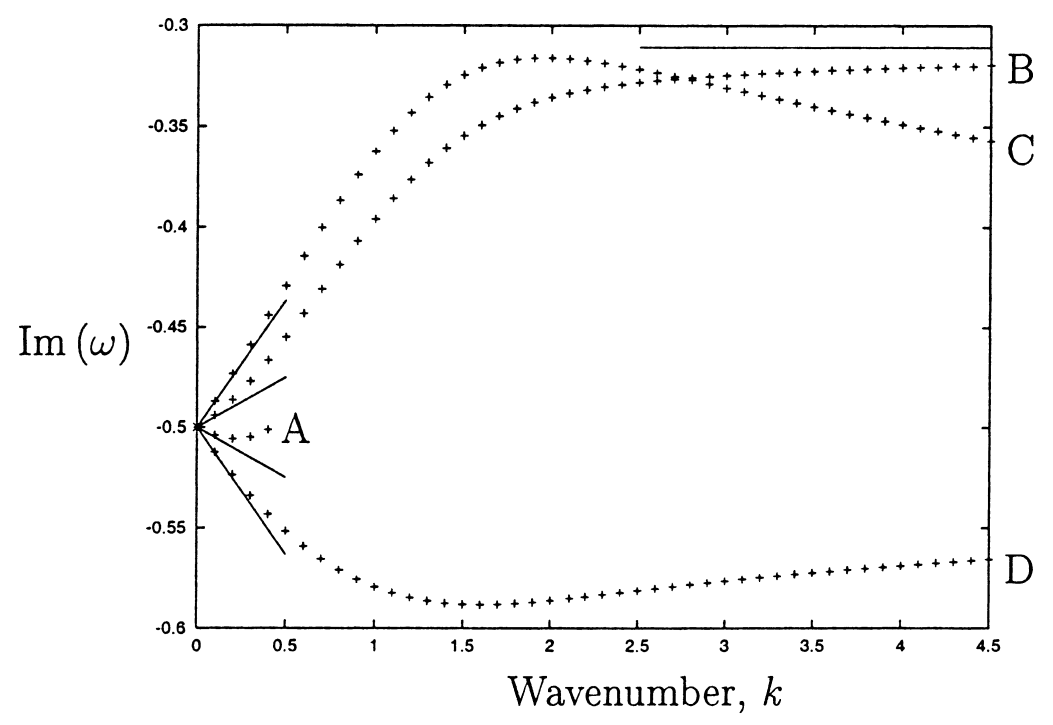

Fig. 8. Growth rates plotted against wavenumber $k$, for an UCM fluid with $W=2$. The four long-wave $(k \rightarrow 0)$ asymptotes shown are given in Section 3, and the short-wave limit for root B is as calculated in Section 4.

Three roots exist for high $k$, but only one (root B) matches the short-wave asymptotics of Section 4. The others do not obey our assumed scaling, but rather have $\omega \sim k+\mathrm{O}(1)$ as $k \rightarrow \infty$. In other words, they become concentrated around the centre-line rather than the wall and represent the stable response of the fluid to small perturbations about a state of rest.

Turning now to the more general problem in which $n \neq 1$, there is again a continuous spectrum of modes, located at $y=y_{0}, 0 \leq y_{0} \leq 1$. Each mode is defined by the requirement that the coefficient

$$
-\mathrm{i} \omega+\mathrm{i} k\left(1-y_{0}^{(n+1) / n}\right)+\tau^{-1}\left(y_{0}\right)
$$

in Eqs. (7)-(9) should vanish, and thus has complex frequency given as

$$
\omega=k\left(1-y_{0}^{(n+1) / n}\right)-\frac{\mathrm{i}}{W}\left[\frac{n+1}{n} y_{0}^{1 / n}\right]^{1-n},
$$

with the corresponding eigenfunction

$$
\psi=\delta\left(y-y_{0}\right)
$$

Fig. 9 shows this continuous spectrum in the $\omega$-plane for $W=2, k=1$ for some illustrative values of $n$. We note that the extreme values of the continuous spectrum are given by $y_{0}=1$, for which

$$
\omega=-\frac{\mathrm{i}}{W}\left(\frac{n+1}{n}\right)^{1-n}
$$

and $y_{0}=0$, for which $\omega=k$, except in the special case $n=1$. 


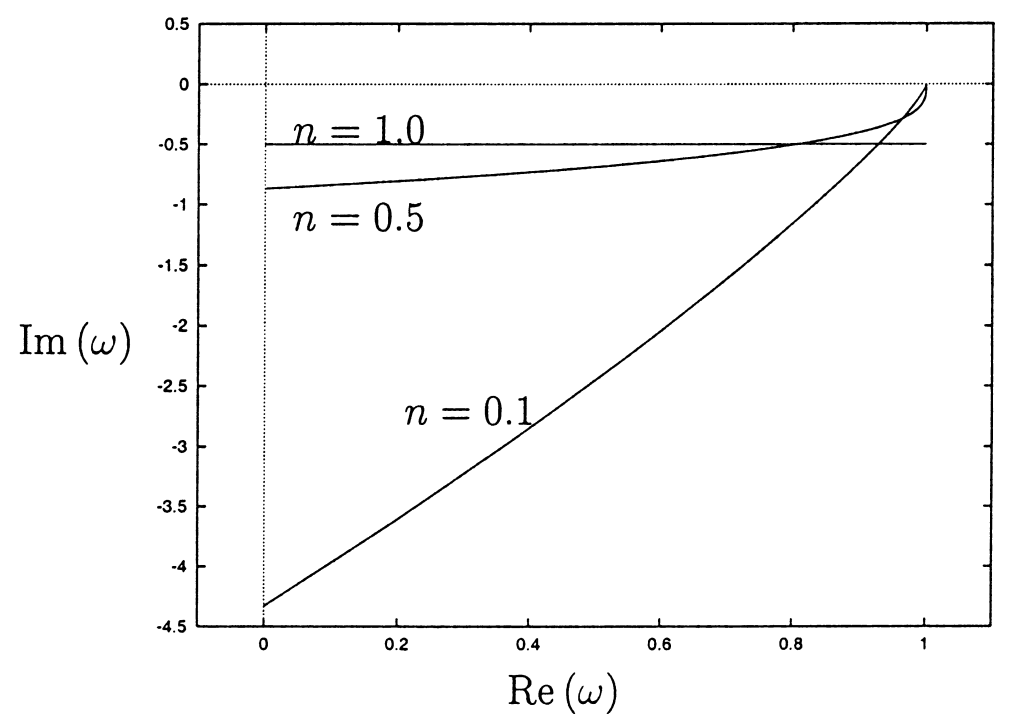

Fig. 9. The continuous spectrum (in the $\omega$-plane) for a WM fluid. $W=2$ and $k=1$; three different values of $n$ are shown.

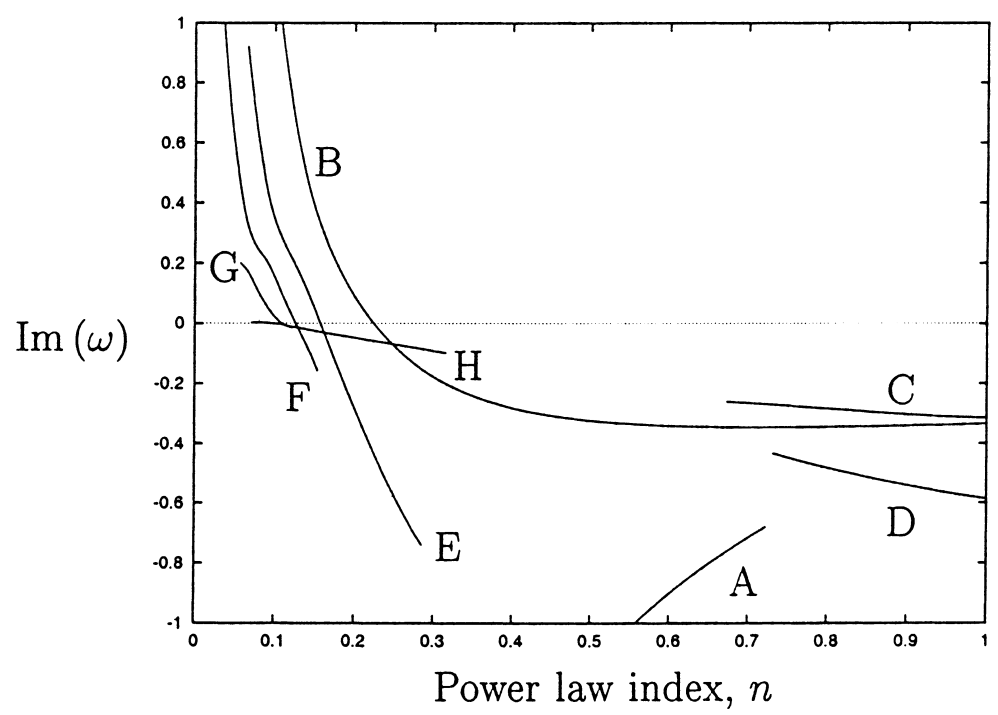

Fig. 10. Growth rate against power-law index of modes for the WM fluid. $W=k=2$.

Because, whenever $n \neq 1$, the continuous spectrum reaches the line of neutral stability, $\operatorname{Im} \omega=0$, analytic continuation of roots can only be of limited value. Roots may cease to exist, and so parameter continuation cannot guarantee to find all acceptable roots of the dispersion relation.

As an illustration, Figs. 10 and 11 show all the roots that we have found for $\omega$ as $n$ varies, when $W=k=2$. There are four roots which are highly unstable for small $n(\mathrm{~B}, \mathrm{E}, \mathrm{F}$ and $\mathrm{G})$, but only one of these (root $\mathrm{B}$ ) continues in $n$ up to $n=1$. A further root, $\mathrm{H}$, is weakly unstable, and disappears at a 


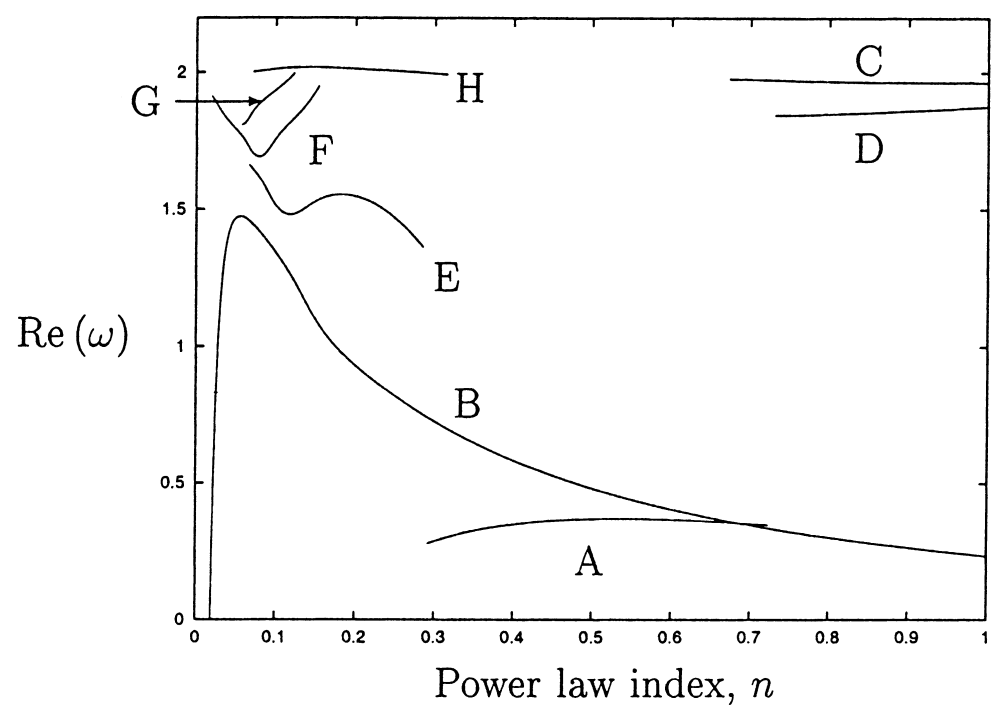

Fig. 11. Real part of the eigenvalues shown in Fig. 10.

critical value of $n \simeq 0.35$. Starting from $n=1$, we know that there are four roots $(\mathrm{A}, \mathrm{B}, \mathrm{C}$ and $\mathrm{D}$, as defined in Fig. 8) but only two of these, $\mathrm{A}$ and $\mathrm{B}$, continue to exist for small $n$. Root $\mathrm{A}$ continues to exist and is stable beyond the scale of Fig. 10 when $n$ is small.

We are unable to continue our calculation to very small $n$. The difficulty is numerical. As $n \rightarrow 0$, the equations become singular, and the stream function derivatives increasingly large. Small errors in the function values are amplified by the integration until they swamp the true value and no root can be found.

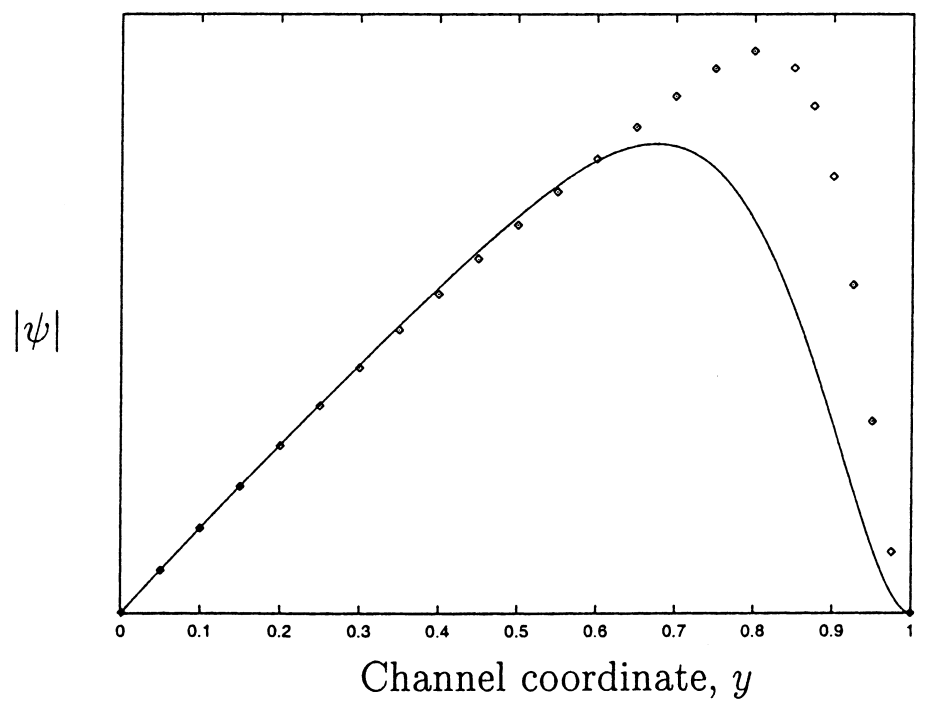

Fig. 12. Unstable eigenfunctions for small values of $n$. The modulus of the stream-function is plotted for root $\mathrm{B}$ at $W=2$, $k=2$. The solid line is for $n=0.2$ and the points for $n=0.1$. 


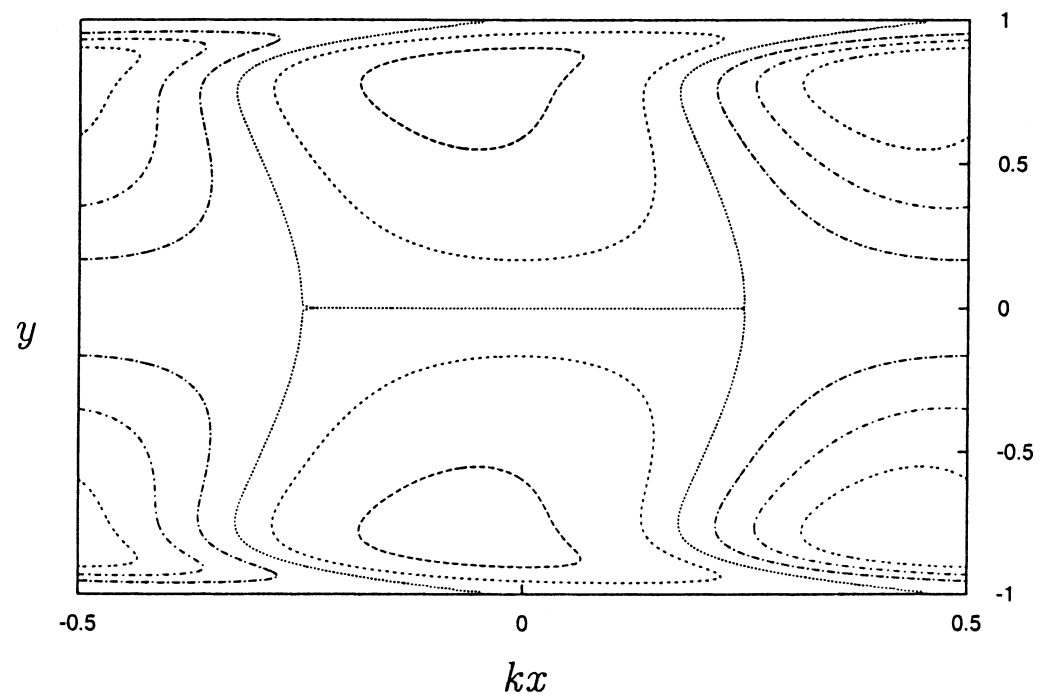

Fig. 13. Streamlines of the perturbation flow for an unstable mode of the WM fluid. $W=2, k=2$, and $n=0.1$ (root B). Note the rapid variation in the boundary layer near the wall. Note also the reversed flow in this region.

In Fig. 12, we show typical unstable eigenfunctions for $n=0.1$ and $n=0.2$. Fig. 13 shows the perturbation streamlines for the $n=0.1$ case. An interesting feature of the streamline plot is the existence of a region of reversed flow in the boundary layer near the wall, so that at a fixed value of $x$, $u(y)$ can have two maxima and two minima. This feature is absent from the stable mode in Fig. 3 . We believe that the reversed flow is a crucial aspect of the instability mechanism as discussed further in Section 6.

To complete the picture of this instability, a study of its wave number-dependence is needed. For no values of $k$ and $W$ did we find any unstable mode for $n>0.3$. The unstable modes are of the greatest interest, and for the values of $n$ that we were able to explore we identify two representative sets of parameter values. The choice $n=0.1, W=2$, is such that short waves are unstable (see Section 4 ) and when $k=2$ we have found five unstable modes (B, E, F, G, H). The choice $n=0.2, W=2$, on the other hand gives short waves that are stable, and has only three unstable modes at $k=2$. We discuss these cases separately.

Figs. 14 and 15 show the imaginary and real parts of the five roots at $n=0.1, W=2$. The most unstable mode (B) corresponds to the short-wave root as $k \rightarrow \infty$ as discussed in Section 4, and to the stable long-wave root as $k \rightarrow 0$ discussed in Section 3. The other four roots cease to exist when the waves become long ( $k$ small), by merging into the continuous spectrum. This result is consistent with our observation in Section 3 that the condition $k W$ sufficiently large is necessary for instability. For the case $n=0.1, W=2$, it is apparent from Fig. 14 that the most unstable disturbance is a short wave. Three of the five roots in Figs. 14 and 15, roots B, E and F, are sufficiently widely spaced to be clearly distinguishable on the plot, but the other two, roots $\mathrm{G}$ and $\mathrm{H}$, are very close together. In fact, they are close to a mode-crossing point, as shown on a larger scale in Figs. 16 and 17. The plots illustrate the richness of phenomena that arise (and the corresponding difficulties of accurate numerical resolution) as $n$ becomes small. 


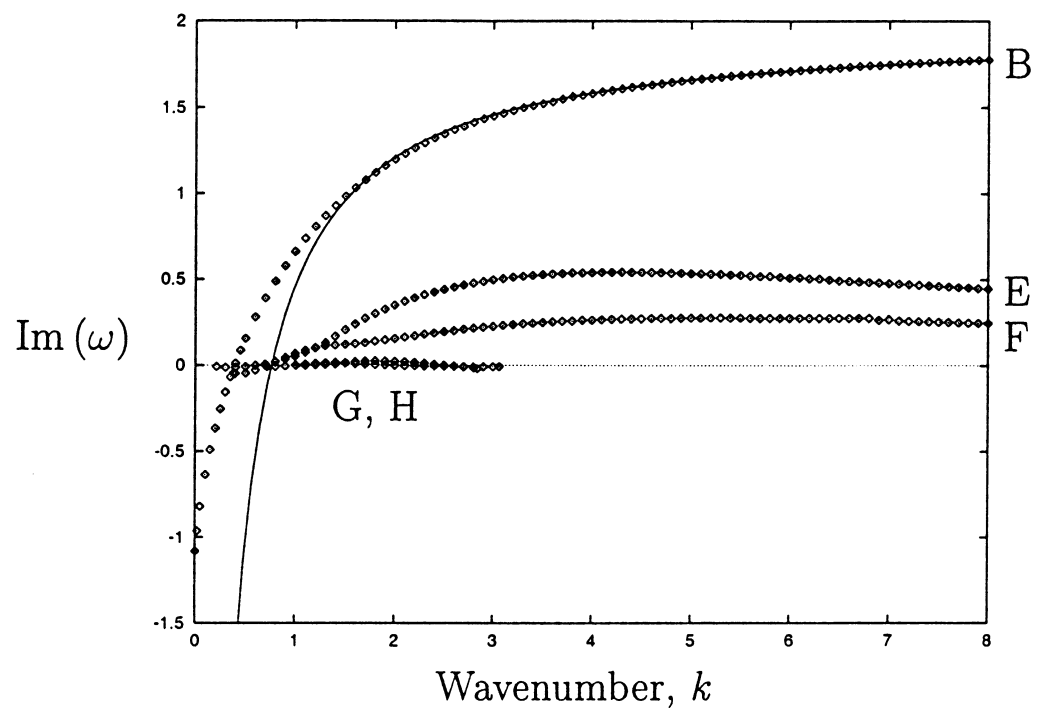

Fig. 14. The wavenumber-dependence of the growth rate of the five unstable roots, for a WM fluid with $W=2$ and $n=0.1$. The numerical results are all shown as points. The solid line is a numerical extrapolation of the most unstable root B as $k \rightarrow \infty$ given by $\operatorname{Im}(\omega)=\operatorname{Im}\left(\omega_{\infty}\right)-1.5 k^{-1}$, where $\omega_{\infty}$ is the short-wave root found in Section 4 .

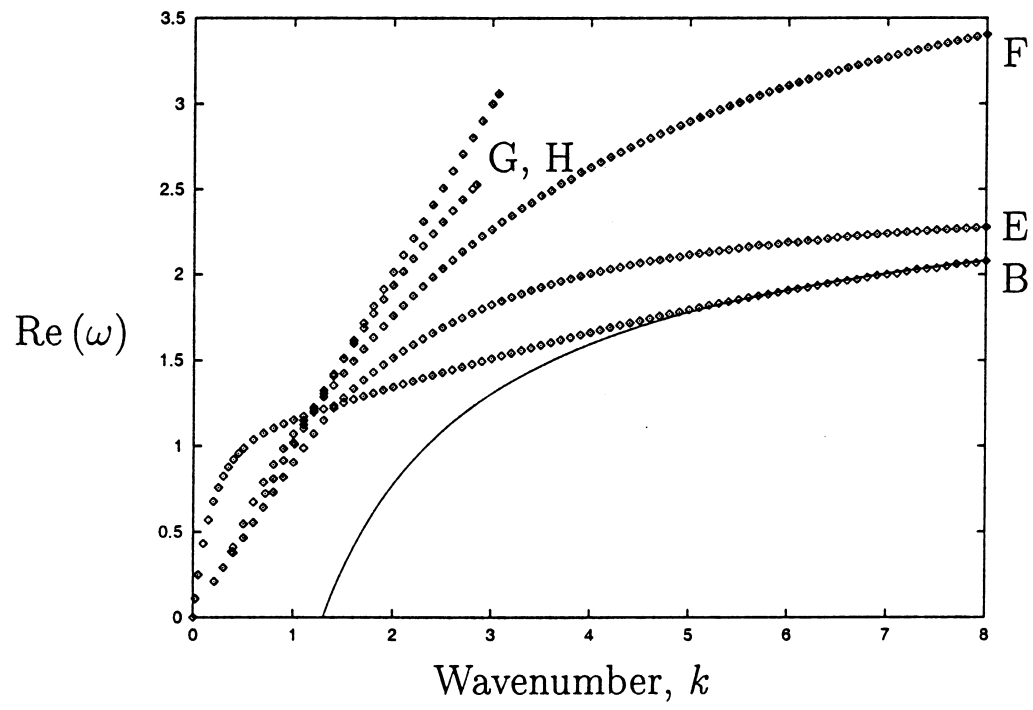

Fig. 15. The real parts of the eigenvalues shown in Fig. 14. The solid line is an extrapolation of the behaviour of root B, the most unstable root, as $k \rightarrow \infty$ matching up with the numerical results for short waves from Section 4.

The numerically less difficult case $n=0.2, W=2$ has only three unstable roots shown in Figs. 18 and 19. In this case, the most unstable disturbance (root B) has $k$ of order unity and restabilises as $k \rightarrow \infty$. The most unstable eigenfunction is shown in Fig. 20. 


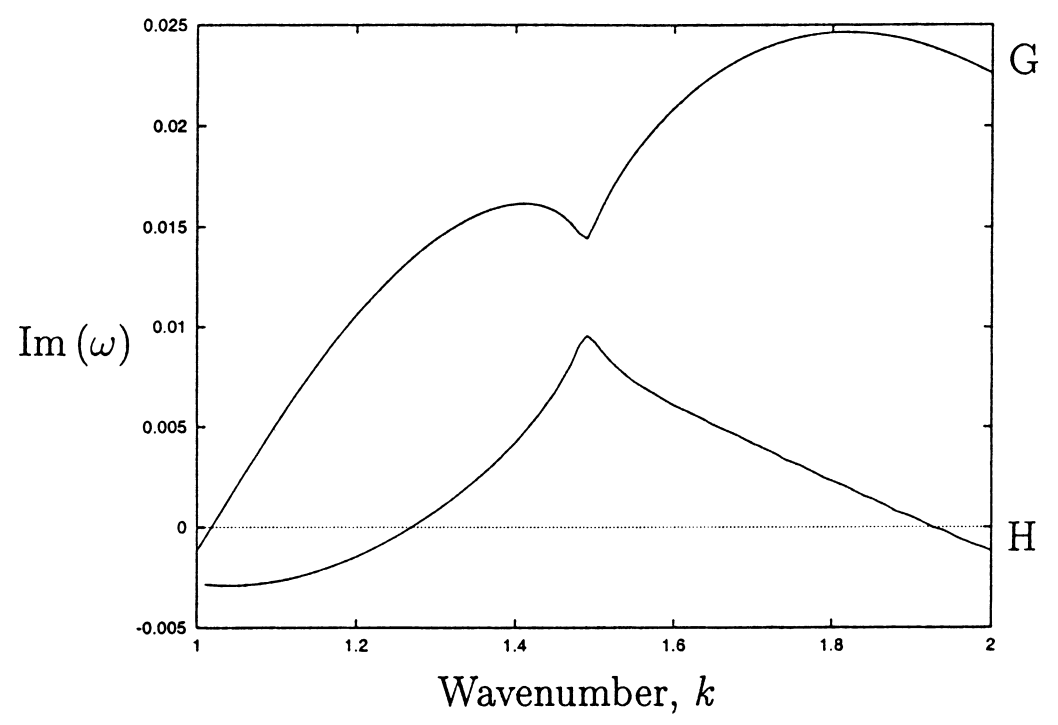

Fig. 16. Close-up of part of Fig. $14 W=2$ and $n=0.1$. The two roots shown, roots $\mathrm{G}$ and $\mathrm{H}$, are close (in parameter-space) to a point where they cross.

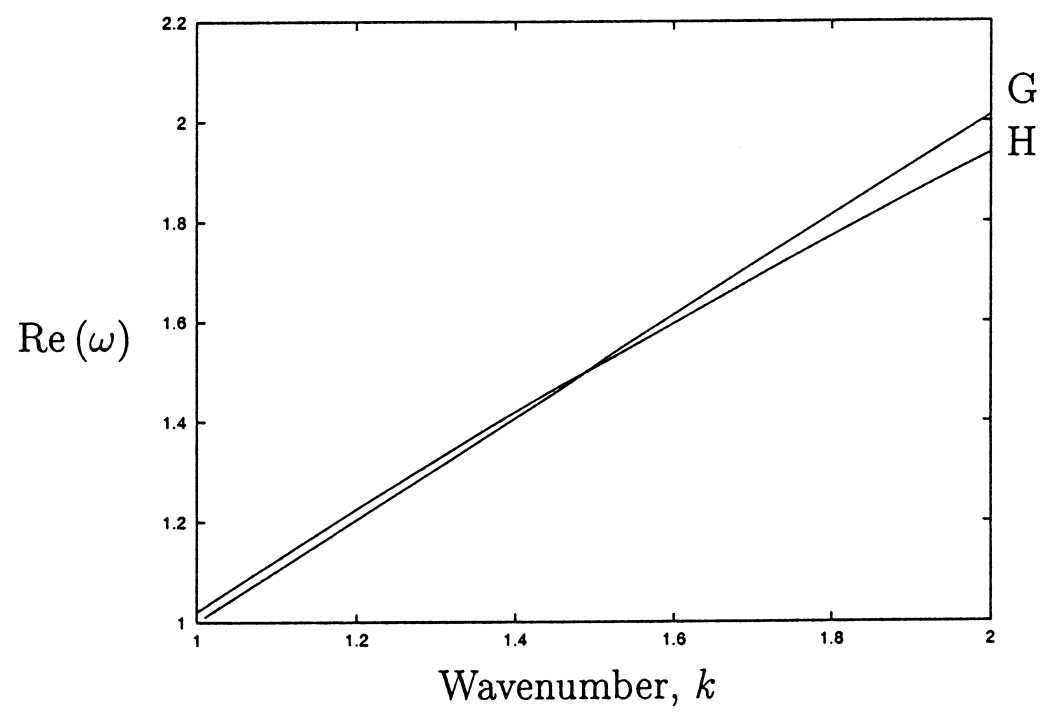

Fig. 17. The behaviour of the real parts of roots $\mathrm{G}$ and $\mathrm{H}$ close to their mode-crossing. This plot shows the real part of the curves shown in Fig. 16.

\section{Instability mechanism}

We have noted that for channel flows of a power-law WM fluid an inertialess instability arises only if the viscosity shear-thins sufficiently (i.e. for which $n$ is sufficiently small) and for disturbances having short or moderate wavelength. We have seen too that if $n$ is very small, several branches of stable and unstable modes exist. This behaviour is very different from the co-extrusion instability [12], and we 


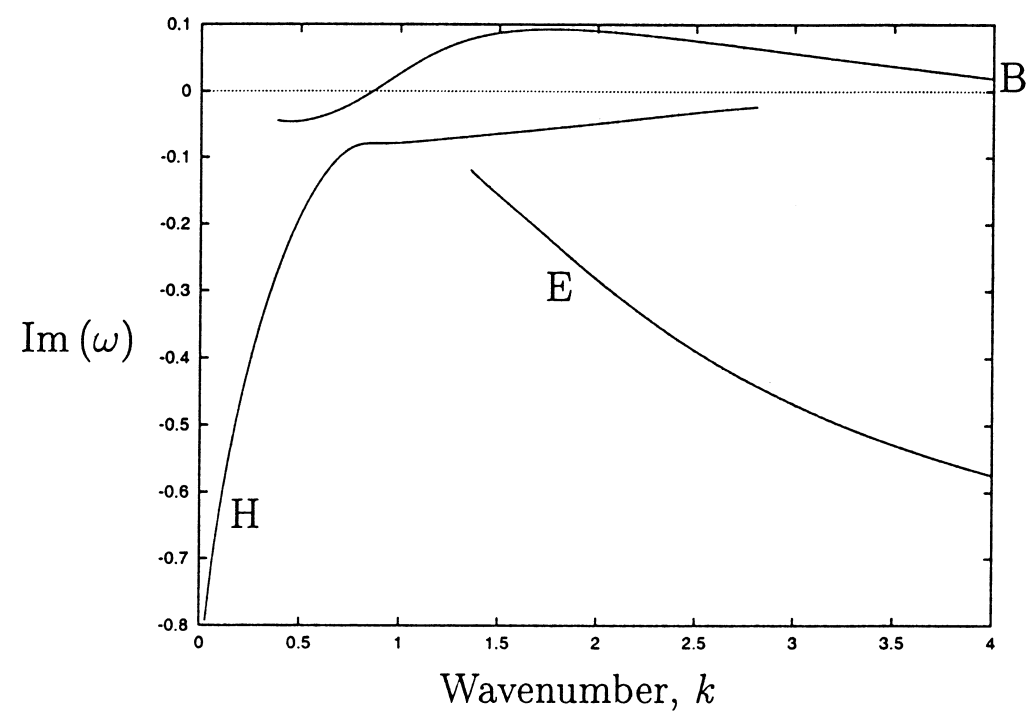

Fig. 18. The wavenumber-dependence of the growth rate of the three roots for a WM fluid with $W=2$ and $n=0.2$.

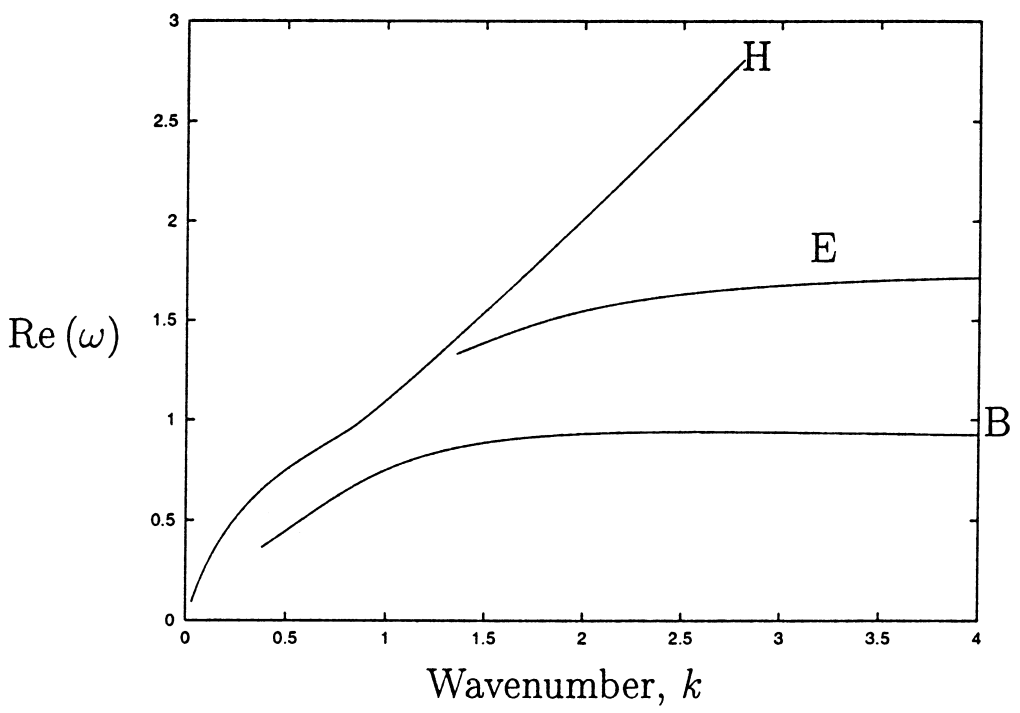

Fig. 19. The wavenumber-dependence of the convective part of the eigenvalue of the three roots for a WM fluid with $W=2$ and $n=0.2$. This plot shows the real part of the curves whose imaginary parts are in Fig. 18.

seek here to identify the small- $n$ mechanism for a mode having growth rate of order unity. A full explanation would involve obtaining a solution of the problem in the singular $n \rightarrow 0$ limit by matched asymptotic expansions; we have not succeeded in obtaining such a solution, nevertheless, some key features may be identified.

We note first that away from the boundary layer where $1-y=\mathrm{O}(n)$, the perturbation flow Deborah number $\omega \tau$ is proportional to $\omega y^{-1 / n} / n$, and this is very large. The material, therefore, behaves like a 


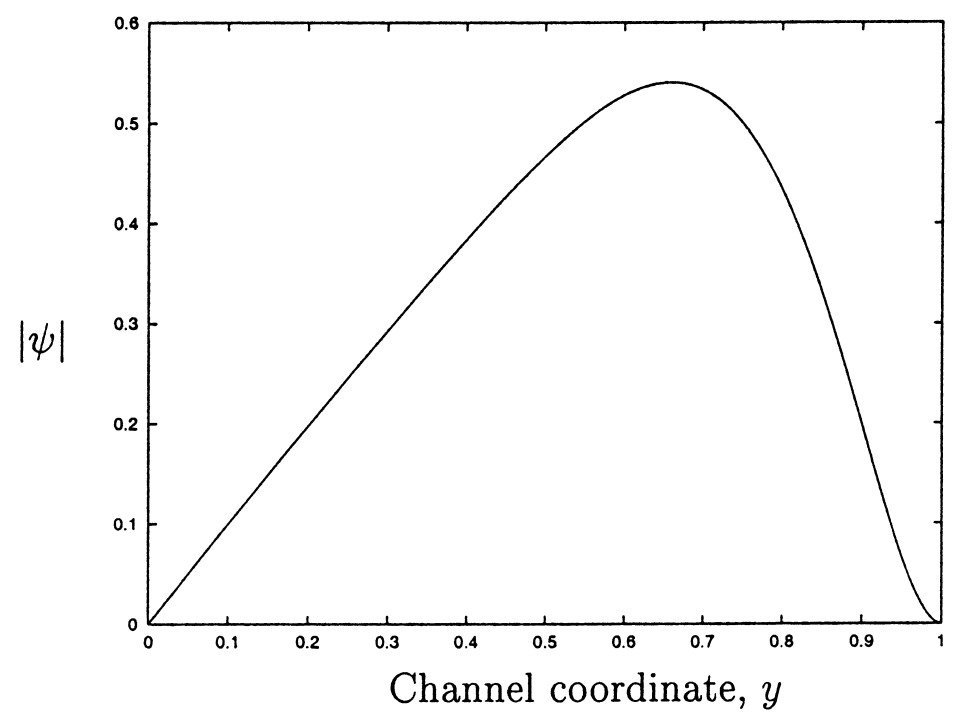

Fig. 20. The eigenfunction for the most unstable mode, root $\mathrm{B}$, for a WM fluid with $n=0.2, W=2$ and $k=1.77$.

solid, and the frequency $\omega$ makes no explicit appearance in the dynamics of the perturbation. On the other hand, close to the wall, $\omega \tau=\omega / n$ is small and so the material is a viscous fluid and again the value of $\omega$ is irrelevant. We conclude that the role of the eigenvalue $\omega$ is to specify the position at which the material changes from fluid to solid. This point occurs close to the edge of the boundary layer where $1-y \sim n(\ln \omega-\ln n)$. It is the appearance of logarithmic terms in $n$ here that have frustated our attempts to provide a full asymptotic solution as $n \rightarrow 0$.

We showed in [1] that for Oldroyd fluids, relative convection of different fluid layers within the channel can stabilise the co-extrusion instability. That issue is irrelevant here, however, for in the interior region the base state velocity profile is plug flow, so no relative convection arises. In the wall layer, the convection term is $k U(y)=k\left(1-y^{1 / n}\right)$, and except for very short waves as discussed in Section 4 , this convection is everywhere small compared with the relaxation rate $y^{1 / n} / n$ and thus, may again be ignored.

The solid-like interior region resists deformation. In consequence, the largest velocities and shear rates associated with the unstable mode being considered lie within and close to the margin of the fluid wall layer as may be seen in Fig. 13. Several conclusions follow. First, we do not expect any difference between varicose and sinuous modes: the boundary condition at $y=0$ is too distant to have an effect. Second, the pattern of normal stresses within the interior is irrelevant: only the normal stresses near the walls can matter. Third, because the mode is localised in the shear-thinned region near the wall it may be difficult to see experimentally, or may itself induce a finite-amplitude stick-slip instability that we have not included in this analysis.

The dynamics of the wall layer are surprisingly complex, involving not only the scales $n$ and $n \ln n$ as identified above, but also $n^{1 / 2}$, which is the scale of the interior flow to which the wall layer should be matched.

In the layer closest to the wall where $1-y$ is comparable with $n$, the zero-Deborah-number perturbation flow, $u(y)$, is necessarily unidirectional at leading order and might, therefore, be expected 
to be simply a multiple of the base flow. That is not the case. Within this layer, $\partial u / \partial x$ has magnitude $\mathrm{i} k u$, and mass conservation gives $\partial v / \partial y$ of the same size. There is a resulting perturbation to $A_{22}$ (which has magnitude 1 in the base state) of size $\mathrm{i} k u$. In other words, the polymer stretch perpendicular to the channel walls is slightly modified by the extension present in the perturbation flow. Now the base shear flow acting on the perturbed $A_{22}$ gives rise to corresponding perturbations of size ikW in $A_{12}$ (and $\mathrm{i} k W^{2}$ in $A_{11}$ ) as would appear in any other steady shear flow. The fluid viscosity in the shearthinned wall layer has magnitude $n$, and because this layer is thin its dynamics are controlled by the shear stress; the normal stress difference $a_{11}-a_{22}$ does not appear. Within an arbitrary constant multiplier for $u$, the vorticity Eq. (6) and the evolution Eq. (8) for the shear stress in the boundary layer reduce to

$$
n u^{\prime}+2 \mathrm{i} k W u=(1+2 \mathrm{i} k W) y^{1 / n} .
$$

Now one solution of this equation is the expected multiple $u=y^{1 / n}$ of the base flow, but there is a second contribution $u=-\mathrm{e}^{2 \mathrm{i} k W(1-y) / n}$ that is oscillatory on a scale $k W / n$. Provided $k W$ exceeds some critical value of about $\pi / 2$, then within the region where $1-y$ is of size $n$ a region of reversed flow is possible. Indeed regions of reversed $u$ may be seen in the unstable eigenfunction plots of Fig. 13, (and also for the short wave limit in Fig. 7 although the balance of terms is slightly different there). If $k W$ is too small, no flow reversal takes place in the boundary layer (e.g. see Fig. 3.)

Further from the wall (in fact, at distances of size $n^{1 / 2}$ the first normal stress difference does enter the dynamical balance, and decays exponentially at a rate $k W / n^{1 / 2}$ ) into the interior solid region. The existence of parasitic growing exponentials is one reason for the numerical difficulties in solving the eigenmode equations for small $n$. One might expect, as sketched in Fig. 21 that the presence of this elastic tension in the perturbed streamlines would act as a stabilising influence, generating a perturbation flow having a direction that would reduce the streamline displacements. But we have seen above that if $k W$ is large enough, flow reversal is possible in the wall layer, and this may give instead a flow which has the opposite sense, and tends to increase the streamline perturbation. This is precisely the feature seen in the unstable streamline plots of Figs. 13 and 7.

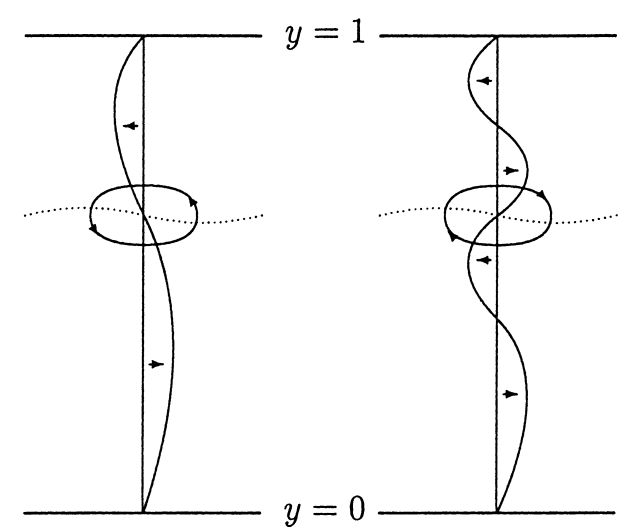

Fig. 21. Sketch of the mechanism of instability. On the left is a stable case: the tension in the streamlines generates a perturbation flow profile as shown that depresses the crests. For the unstable case (diagram on the right) flow reversal in the wall layer amplifies the perturbation to the streamlines. 
We suggest then that it is the driving force supplied by the elastic normal stresses, coupled with the counter-intuitive oscillations of the boundary layer flow when $n \ll 1$ and $k W>1$ that provides the destabilising mechanism. The value of $\omega$ must be chosen correctly so as to match the phase of the oscillatory fluid wall layer to the interior solid region.

\section{Conclusions}

We have found, for a strongly shear-thinning WM fluid, a purely elastic instability in channel flow. At least one linearly unstable mode is concentrated in the boundary layer near the wall. Its mechanism, which will operate also in pipe flows, involves both normal stresses close to the wall and, in a subtle way, the constitutive assumption for the WM model that the fluid relaxation time depends upon the local instantaneous shear rate. This mechanism is fundamentally different from that of the co-extrusion instability or of the curved-streamline instability that arises in Taylor-Couette flow.

Two questions stem naturally from this work. The first is computational: in a full numerical simulation of channel flow of a power-law WM fluid, what form of finite amplitude instability is obtained? The second is physical: for an elastic fluid, presumably a polymer melt, which shear-thins sufficiently, is the instability in channel flow that we have found theoretically observed in practice? This is a severe test of the WM constitutive assumption. If this hydrodynamic instability is observed, it has important implications for polymer processing.

\section{Acknowledgements}

This work was assisted by the EU Human Capital and Mobility Programme Grant No. CHRX-LT930200. HJW was supported by a Research Studentship from the EPSRC.

\section{References}

[1] R.G. Larson, Instabilities in viscoelastic flows, Rheol. Acta 31(3) (1992) 213-263.

[2] H.J. Wilson, J.M. Rallison, Instability of channel flows of elastic liquids having continuously stratified properties. J. Non-Newtonian Fluid Mech., 1999, in press.

[3] E.J. Hinch, O.J. Harris, J.M. Rallison, The instability mechanism for two elastic liquids being coextruded, J. NonNewtonian Fluid Mech. 43(2/3) (1992) 311-324.

[4] J.L. White, A.B. Metzner, Development of constitutive equations for polymeric melts and solutions, J. Appl. Polym. Sci. 7 (1963) 1867-1889.

[5] V.A. Gorodtsov, A.I. Leonov, On a linear instability of a plane parallel Couette flow of viscoelastic fluid, J. Appl. Math. Mech. 31 (1967) 310.

[6] P.J. Carreau, Rheological equations from molecular network theories, Ph.D. thesis, University of Wisconsin, Madison, 1968.

[7] H.J. Wilson, Shear flow instabilities in viscoelastic fluids, Ph.D. thesis, University of Cambridge, 1998.

[8] H.J. Wilson, M. Renardy, Y.Y. Renardy, Structure of the spectrum in zero Reynolds number shear flow of the UCM and Oldroyd-B liquids, J. Non-Newtonian Fluid Mech., submitted for publication.

[9] K.P. Chen, Interfacial instability due to elastic stratification in concentric coextrusion of two viscoelastic fluids, J. NonNewtonian Fluid Mech. 40(2) (1991) 155-176. 
[10] E.S.G. Shaqfeh, Purely elastic instabilities in viscometric flows, Ann. Rev. Fluid Mech. 28 (1996) $129-186$.

[11] T.C. Ho, M.M. Denn, Stability of plane Poiseuille flow of a highly elastic liquid, J. Non-Newtonian Fluid Mech. 3(2) (1978) 179-195.

[12] H.J. Wilson, J.M. Rallison, Short wave instability of co-extruded elastic liquids with matched viscosities, J. NonNewtonian Fluid Mech. 72(2/3) (1997) 237-251. 